\title{
Partition function zeroes of a self-dual Ising model
}

\author{
Wentao T. Lu and F. Y. Wu \\ Department of Physics \\ Northeastern University, Boston, Massachusetts 02115
}

\begin{abstract}
We consider the Ising model on an $M \times N$ rectangular lattice with an asymmetric self-dual boundary condition, and derive a closed-form expression for its partition function. We show that zeroes of the partition function are given by the roots of a polynomial equation of degree $2 M-1$, which trace out certain loci in the complex temperature plane. Particularly, it is shown that (a) real solutions of the polynomial equations always lead to zeroes on the unit circle and a segment of the negative real axis, and (b) all temperature zeroes lie on two circles in the limit of $M \rightarrow \infty$ for any $N$. Closed-form expressions of the loci as well as the density of zero distributions in the limit of $N \rightarrow \infty$ are derived for $M=1$ and 2. In addition, we explain the reason of, and establish the criterion for, partition function zeroes of any self-dual spin model to reside precisely on the unit circle. This elucidates a recent finding in the case of the self-dual Potts model.
\end{abstract}

$05.50 .+\mathrm{q}$ 


\section{INTRODUCTION}

One of the most remarkable results in statistical mechanics is the circle theorem of Yang and Lee [1] who in 1952 established that zeroes of the partition function of the ferromagnetic Ising model always lie on the unit circle in the complex $e^{-2 L}$ plane, where $L=H / k T$ is the reduced magnetic field variable. In 1964 Fisher 20 pointed out that it is also meaningful to consider zeroes of the Ising partition function in the complex temperature plane, in which the zeroes reside on a boundary at which the free energy becomes non-analytic. Particularly, he showed that temperature zeroes of the square lattice Ising model lie on two circles in the thermodynamic limit. Since then the consideration of the partition function zeroes has become a power tool in analyzing lattice models. The temperature zeroes of the Ising model [3, [4] as well as the Potts model [5] have been investigated. However, studies to this date have been confined mostly to results in the thermodynamic limit [2.3] and/or numerical analyses [4.5.5]. Very little progress has been made for finite lattices, except in one dimension [6], in which the locations of zeroes are determined in a closed-form and analyzed algebraically.

In this paper we study the partition function zeroes of the Ising model for a finite rectangular lattice with an asymmetric self-dual boundary condition. This study is motivated by a recently discovered numerical evidence in the case of self-dual Potts models [5] that many partition function zeroes lie precisely on the unit circle. Here, we explain why this is the case, and establish more generally the criterion for zeroes to occur on the unit circle for self-dual lattice models. Particularly, for the Ising model considered here, we show that all zeroes lie on two circles in the limit of $M \rightarrow \infty$ and any $N$. We also obtain closed-form expressions for the loci of zeroes for $M=1,2$, as well as the density of zero distribution in the limit of $N \rightarrow \infty$.

\section{A SELF-DUAL RECTANGULAR LATTICE}

Consider a rectangular Ising lattice $\mathcal{L}$ of $M$ rows and $N$ columns with periodic boundary conditions in the horizontal direction. Let the nearest-neighbor interaction be $-k T K$. Further, introduce a boundary condition that all sites on one of the two horizontal boundaries, say, the lowermost one, interact with an additional site with the same interaction $-k T K$. Thus, there are altogether $M N+1$ sites and $2 M N$ edges. Note that the boundary condition is "asymmetric" in the horizontal and vertical directions.

Topologically, $\mathcal{L}$ assumes the form of a "wheel" consisting of $N$ spokes and $M$ concentrate circles. An example of $\mathcal{L}$ is shown in fig. 1(a) where the circumference of circle corresponds to the "horizontal" direction. We point out that the lattice $\mathcal{L}$ is self-dual, which is an important premise of our consideration.

The high-temperature expansion of the partition function assumes the form

$$
Z_{M, N}=2^{M N+1}(\cosh K)^{2 M N} G_{\mathcal{L}}(z)
$$

where $z=\tanh K$ and

$$
G_{\mathcal{L}}(z)=1+\sum_{\text {c.p. } \in \mathcal{L}} z^{b} .
$$


Here, the summation in (2) is over all closed polygonal configurations that can be drawn on $\mathcal{L}$, and $b$ is the number of edges contained in each polygonal configuration.

To facilitate our considerations, we expand the center point of the wheel in Fig. 1(a) into a circle as shown in Fig. 1(b), and associate edge weights 1 to the $M$ newly added edges. This transforms the lattice $\mathcal{L}$ into $\mathcal{L}^{*}$. A moment's reflection [7] shows that we have the relation

$$
G_{\mathcal{L}}(z)=\frac{1}{2} G_{\mathcal{L}^{*}}(z)
$$

It follows that the partition function (11) is computed if we can evaluate $G_{\mathcal{L}}^{*}(z)$.

The lattice $\mathcal{L}^{*}$ is an $(M+1) \times N$ rectangular lattice with a periodic boundary condition in the horizontal direction. All edges of $\mathcal{L}^{*}$ carry the weight $z$ except those on the innermost circle which carry the weight 1 . Following Kasteleyn [8] we can express $G_{\mathcal{L}^{*}}(z)$ as a Pfaffian which is, in turn, the square root of a determinant. The procedure, which is standard, has been described in details by McCoy and Wu [9, 10, and leads to

$$
G_{\mathcal{L}^{*}}(z)=\left[\prod_{n=1}^{N} \operatorname{det}\left|B_{M}(\phi)\right|\right]^{1 / 2},
$$

where $B_{M}(\phi)$ is a $4(M+1) \times 4(M+1)$ matrix given by

$$
B_{M}(\phi)=\left(\begin{array}{ccccc}
B(z) & B_{+}(z) & & & \\
B_{-}(z) & B(z) & B_{+}(z) & & \\
\vdots & \vdots & \ddots & \vdots & \vdots \\
& & B_{-}(z) & B(z) & B_{+}(z) \\
& & & B_{-}(z) & B(1)
\end{array}\right) .
$$

Here, the product in (幽 is taken over the $N$ values

$$
\phi=(2 n-1) \pi / N, \quad n=1,2, \cdots N,
$$

and $B(z)$ and $B_{ \pm}(z)$ are $4 \times 4$ matrices given by (A5) in the Appendix. For completeness, an outline of the derivation of (4) is given in the Appendix. Note that $\phi \neq 0, \pi$. The

substitution of (5) into (3) and (11) now expresses the partition function $Z_{M, N}$ in terms of a $4(M+1) \times 4(M+1)$ determinant.

\section{EVALUATION OF THE PARTITION FUNCTION}

In this section we evaluate the determinant $\operatorname{det}\left|B_{M}(\phi)\right|$ in (身) using an approach somewhat different from that of [9].10], and present the solution in a form more suitable for our purposes.

For brevity we write $B_{M} \equiv \operatorname{det}\left|B_{M}(\phi)\right|$. The determinant $B_{M}$ is bilinear in the two non-vanishing elements $z$ in $B_{+}(z)$ in the first row and $-z$ in $B_{-}(z)$ in the first column of (5). Explicitly, the bilinear expansion is

$$
B_{M}=\operatorname{det}|B(z)| \cdot B_{M-1}+z\left[B_{M}\right]_{1 ; 6}-z\left[B_{M}\right]_{6 ; 1}+z^{2}\left[B_{M}\right]_{1,6 ; 1,6}
$$


where $\left[B_{M}\right]_{i ; k}$ is the determinant of the matrix $B_{M}(\phi)$ with the $i$ th row and $k$ th column removed, and $\left[B_{M}\right]_{(i j ; k \ell)}$ is similarly defined. We have the following lemma whose proof is elementary and will not be given:

Lemma: For any any $m \times n$ matrix $A_{m n}$ and $(N-m) \times(N-n)$ matrix $C_{N-m, N-n}$, we have the identity

$$
\operatorname{det}\left|\begin{array}{cc}
A_{m n} & 0 \\
0 & C_{N-m, N-n}
\end{array}\right|=\delta_{m, n} \cdot \operatorname{det}\left|A_{m n}\right| \cdot \operatorname{det}\left|C_{N-m, N-n}\right|,
$$

where $\delta$ is the Kronecker delta function.

It follows from the lemma that we have

$$
\begin{gathered}
{\left[B_{M}\right]_{1 ; 6}=\left[B_{M}\right]_{6 ; 1}=0,} \\
{\left[B_{M}\right]_{1,6 ; 1,6}=\operatorname{det}|B(z)|_{1,1} \cdot\left[B_{M-1}\right]_{2 ; 2} .}
\end{gathered}
$$

Writing $D_{M} \equiv\left[B_{M}\right]_{2,2}$ and evaluating $\operatorname{det}|B(z)|$ and $\left[B_{M}\right]_{1,6 ; 1,6}$ explicitly, (7) becomes

$$
B_{M}=a B_{M-1}+b D_{M-1},
$$

with

$$
a=1+z^{2}-2 z \cos \phi, \quad b=2 i z^{3} \sin \phi .
$$

In a similar fashion we expand $D_{M}$ and obtain

$$
D_{M}=c B_{M-1}+d D_{M-1},
$$

with

$$
c=-2 i z \sin \phi, \quad d=z^{3}\left(1+z^{2}+2 z \cos \phi\right) .
$$

Both (11) and (13) hold for any $M \geq 1$ with the boundary condition

$$
B_{0}=\operatorname{det}|B(1)|=2-2 \cos \phi, \quad D_{0}=\operatorname{det}\left|B_{0}(1)\right|_{2,2}=-2 i \sin \phi .
$$

Introducing generating functions

$$
B(\tau)=\sum_{M=0}^{\infty} B_{M} \tau^{M}, \quad D(\tau)=\sum_{M=0}^{\infty} D_{M} \tau^{M}
$$

and multiplying both sides of (11) and (13) by $\tau^{M}$, followed by summing over from $M=1$ to $\infty$, one obtains

$$
\begin{aligned}
& B(\tau)-B_{0}=\tau[a B(\tau)+b D(\tau)] \\
& D(\tau)-D_{0}=\tau[c B(\tau)+d D(\tau)] .
\end{aligned}
$$

This yields 


$$
B(\tau)=\frac{B_{0}(1-d \tau)+D_{0} b \tau}{1-(a+d) \tau+(a d-b c) \tau^{2}}
$$

It is then a simple matter to obtain an explicit expression for $B_{M}$ by expanding the righthand side of (18) and comparing with (16), after first carrying out a partial fraction. After some algebra, this leads to the expression

$$
B_{M}=(2-2 \cos \phi)\left[z\left(1-z^{2}\right)\right]^{M}\left[\frac{\sinh [(M+1) t(\phi)]-s^{-1} \sinh [M t(\phi)]}{\sinh t(\phi)}\right],
$$

where

$$
\begin{aligned}
& 2 \cosh t(\phi)=s+s^{-1}-y \\
& s \equiv s(K)=e^{2 K} \operatorname{coth} K, \quad y=2(1+\cos \phi) .
\end{aligned}
$$

Substituting (19) into (44) and (3), we then obtain from (11) the following closed-form expression for the partition function:

$$
Z_{M, N}=2[2 \sinh 2 K]^{M N / 2} \prod_{n=1}^{N}\left[\frac{\sinh [(M+1) t(\phi)]-s^{-1} \sinh [M t(\phi)]}{\sinh t(\phi)}\right]^{1 / 2} .
$$

Here, use has been made of the identity [11]

$$
\prod_{n=1}^{N}\left[2-2 \cos \left(\frac{2 n-1}{N} \pi\right)\right]=4 \text {. }
$$

We remark that we find also

$$
D_{M}=-2 i\left[z\left(1-z^{2}\right)\right]^{M} \frac{\sin \phi}{\sinh t(\phi)}[\sinh [(M+1) t(\phi)]+s(-K) \sinh [M t(\phi)]] .
$$

\section{ZEROES OF THE PARTITION FUNCTION}

¿From the closed-form expression (22) we find the partition function to vanish at

$$
s=\frac{\sinh [M t(\phi)]}{\sinh [(M+1) t(\phi)]}
$$

a result which holds for all $M \geq 1$ and $\phi$ equal to the $N$ values given by (6). Note that, since only $\cos \phi$ appears in the expression, distinct zeroes are obtained by using only $n=1,2, \cdots,[(N+1) / 2]$, where $[(N+1) / 2]$ is the integer part of $(N+1) / 2$, equal to $N / 2$ for $N=$ even and $(N+1) / 2$ for $n=$ odd. It should also be noted that the numerator and denominator of (25) contain a common factor $\sinh t(\phi)$ which cancels, resulting in polynomials of $2 \cosh t(\phi)$, or $s+s^{-1}-y$, of degrees $M-1$ and $M$, respectively. Equating both sides of (25) after multiplying by the denominator, one finds that the two terms of the order of $s^{-M}$ cancel, so that (25) assumes the form 


$$
P_{2 M-1}(s)=0, \quad n=1,2, \cdots,\left[\frac{N+1}{2}\right]
$$

where $P_{2 M-1}(s)$ is a polynomial of degree $2 M-1$ in $s$. Explicitly, we find

$$
\begin{array}{ll}
2 \cosh t(\phi)-s^{-1}=0, & M=1 \\
4 \cosh ^{2} t(\phi)-2 s^{-1} \cosh t(\phi)-1=0, & M=2 \\
8 \cosh ^{3} t(\phi)-4 s^{-1} \cosh ^{2} t(\phi)-4 \cosh t(\phi)+s^{-1}=0, & M=3,
\end{array}
$$

which yield, after using (20),

$$
\begin{array}{lc}
s-y=0, & M=1 \\
s^{3}-2 y s^{2}+y^{2} s-y=0, & M=2 \\
s^{5}-3 y s^{4}+3 y^{2} s^{3}-\left(2 y+y^{3}\right) s^{2}+2 y^{2} s-y=0, & M=3 .
\end{array}
$$

Note that no closed-form expression exists for the roots for $M \geq 3$.

Guided by results of a recent study [5], we now introduce the variable

$$
x=\left(e^{2 K}-1\right) / \sqrt{2}
$$

so that from (21) we have

$$
s=\sqrt{2}\left(x+x^{-1}\right)+3 .
$$

This says that if $x$ is a root of (26), then $x^{-1}$ is also a root. Furthermore, since $\left|x+x^{-1}\right| \leq 2$ if, and only if, $x$ is on the unit circle $|x|=1$, all real solutions of (26) in the regime

$$
3-2 \sqrt{2} \leq s \leq 3+2 \sqrt{2}
$$

lead to solutions on $|x|=1$. In fact, more generally, we have the following theorem:

Theorem 1: Let $P_{L}(x)$ be a polynomial of degree $L$ in $x$ satisfying the reciprocal relation

$$
P_{L}(x)=x^{L} P_{L}\left(x^{-1}\right) .
$$

Then we have (i) $P_{L}(x)$ can be rewritten as a polynomial in a new variable $w$ with

$$
\begin{aligned}
& P_{L}(x)=x^{L / 2} Q_{L / 2}(w), \quad w=x+x^{-1}, \quad L=\text { even } \\
& =x^{L / 2} Q_{L}(w), \quad w=x^{1 / 2}+x^{-1 / 2}, \quad L=\text { odd },
\end{aligned}
$$

where $Q_{L}(w)$ is a polynomial of degree $L$ in $w$.

(ii) Roots of $P_{L}(x)=0$ are on the unit circle $|x|=1$ if, and only if, the corresponding roots of the polynomial $Q(w)=0$ are real and in the regime $-2 \leq w \leq 2$.

Proof:

Property (i) follows from the elementary facts that (a) the reciprocal relation (34) implies $x$ and $x^{-1}$ appear symmetrically in the $Q$ polynomials in the combination of $X_{n}=x^{n}+x^{-n}$, where $n$ is an integer for $L$ even and a half-odd integer for $L$ odd, and (b) $X_{n}$ is a polynomial of $w$. Property (ii) follows immediately. Q.E.D. 
A consequence of Theorem 1 is that zeroes of the partition function of self-dual spin models, which by definition satisfy the reciprocal relation (34) in an appropriate dual variable $x$, reside precisely on the unit circle $|x|=1$ for all real roots of the $Q$ polynomial in $|w| \leq 2$. This explains the numerical findings of [5] that a large number of zeroes of self-dual Potts partition functions are located on the unit circle.

For the self-dual Ising model on $\mathcal{L}$, we have further:

Theorem 2: (i) All real roots of the $[(N+1) / 2]$ polynomial equations $P_{2 M-1}(s)=0$ are bounded by

$$
-1<s_{\min } \leq s \leq s_{\max }<3+2 \sqrt{2}
$$

where the bounds $s_{\min }$ and $s_{\max }$ depend on $M$.

(ii) Zeroes in the $x$-plane corresponding to real $s$ lie either on the negative real axis

$$
-(\sqrt{2}+1)<x_{-}\left(s_{\min }\right) \leq x \leq x_{+}\left(s_{\min }\right)<-(\sqrt{2}-1)<0, \quad s_{\min } \leq s \leq 3-2 \sqrt{2},
$$

or on the horseshoe segment $\theta_{0}$ to $2 \pi-\theta_{0}$ on the unit circle

$$
|x|=1, \quad 3-2 \sqrt{2} \leq s \leq s_{\max } .
$$

Here, $x_{+}(s) x_{-}(s)=1$ with

$$
\begin{aligned}
x_{ \pm}(s) & =\left(s-3 \pm \sqrt{s^{2}-6 s+1}\right) / 2 \sqrt{2}, \\
\theta_{0} & =\cos ^{-1}\left[\left(s_{\max }-3\right) / 2 \sqrt{2}\right] .
\end{aligned}
$$

Note that the horseshoe segment becomes a circle $\left(\theta_{0}=0\right)$ if $s_{\max }=3+2 \sqrt{2}$.

(iii) Zeroes in the $x$-plane corresponding to $|s|=1$ lie on the circle $|x+\sqrt{2}|=1$. Proof:

(i) We first show that no root exists for $s>s_{0} \equiv 3+2 \sqrt{2}$. Assume the contrary, namely, a solution $s>s_{0}$ exists. Then by $(20)$ we have

$$
\begin{aligned}
\cosh t(\phi) & =\frac{1}{2}\left(s+s^{-1}\right)-(1+\cos \phi) \\
& \geq \frac{1}{2}\left(s_{0}+s_{0}^{-1}\right)-(1+\cos \phi) \\
& =2+\cos \phi \\
& >1 .
\end{aligned}
$$

It follow that $t(\phi)$ is real and (25) gives $s<1$ which contradicts the assumption of $s>s_{0}=$ $3+2 \sqrt{2}$. Hence no real root exists for $s>s_{0}$.

Likewise, for $s<0$ solution of (26) we deduce

$$
\cosh t(\phi)<-1
$$

for which $t(\phi)$ must assume the form

$$
t(\phi)=r+i(2 n+1) \pi,
$$

where $r$ is the real part and $n$ is an integer. Then (25) leads to 


$$
s=\frac{-\sinh M r}{\sinh (M+1) r},
$$

showing that negative solutions of (26) always lie in the interval $(-1,0)$. This completes the proof of (i).

(ii) For $-1<s_{\min }<s \leq 3-2 \sqrt{2}$, one solves $x=x_{ \pm}(s)$ from (32). This leads to (37) where we have used $x_{ \pm}(-1)=-(\sqrt{2} \mp 1)$. For $3-2 \sqrt{2} \leq s \leq s_{\max }$, one writes $x=e^{i \theta}$ and solves for $\theta$ from (32). This leads to the result (38) with $\theta_{0}$ given by (39).

(iii) It is straightforward to show that $|s|=1$ by substituting $x=-\sqrt{2}+e^{i \beta}$ into (32) for any real $\beta$. Q.E.D.

\section{LOCI OF ZEROES}

The loci of zeroes can be explicitly determined for $M=1,2$ and in the limit of $M \rightarrow \infty$. Consider first the case of $M=1$.

For $M=1(28)$ gives

$$
s=2(1+\cos \phi),
$$

so that $s_{\min }=0$ and $s_{\max }=4$. It follows that the zeroes are either on the line segment $x=[-\sqrt{2},-1 / \sqrt{2}]$ or on the horseshoe segment of $|x|=1$ with $\theta_{0}=\cos ^{-1}(1 / 2 \sqrt{2})=57.3^{\circ}$. A plot of the loci obtained for $N=4,000$ is shown in Fig. 2. In the limit of large $N$, the zeroes are distributed uniformly in $\phi$ with a density $\rho(\phi)=1 / 2 \pi$. Combining (44), (32) and $x=e^{i \theta}$, we have

$$
\cos \phi=\sqrt{2} \cos \theta-1 / 2
$$

and find that the zeroes distribute on the horseshoe segment with a density

$$
\rho(\theta)=\frac{1}{2 \pi} \frac{d \phi}{d \theta}=\frac{1}{\sqrt{2} \pi} \frac{\sin \theta}{\sin \phi}
$$

which diverges at $\theta_{0}$ as $\left(\theta-\theta_{0}\right)^{-1 / 2}$.

For $M=2$ the cubic equation (29) yields three roots

$$
s_{n}=\frac{2 y}{3}+\omega^{n} \sqrt[3]{Q+\sqrt{\Delta}}+\omega^{2 n} \sqrt[3]{Q-\sqrt{\Delta}}, \quad n=0,1,2
$$

where

$$
\omega=e^{i 2 \pi / 3}, \quad Q=\frac{y}{2}\left(1-\frac{2 y^{2}}{27}\right), \quad \Delta=\frac{y^{2}}{4}\left(1-\frac{4 y^{2}}{27}\right) .
$$

Now $s_{0}$ is always real and assumes the minimum value $s=0$ at $y=0$. In addition, $s_{1,2}$ are real for $\Delta<0$ or $3 \sqrt{3} / 2<y<4$. It follows that we have $s_{\min }=0$. A more detailed analysis shows that we have $s_{\max }=8(1+\cos \alpha) / 3$, where $\alpha=\frac{1}{3} \cos ^{-1}(-5 / 32)$. These lead to zeroes in the $x$-plane on the negative real segment $[-\sqrt{2},-1 / \sqrt{2}]$ and a horseshoe segment of the unit circle from $\theta_{0}$ to $2 \pi-\theta_{0}$, with $\theta_{0}=\cos ^{-1}[(8 \cos \alpha-1) / 6 \sqrt{2}]=47.7^{\circ}$. A plot of the 
zeroes for $N=2,000$ is shown in Fig. 3. The density of zeroes in the limit of $N \rightarrow \infty$, which can be found accordingly, is given as the superposition of the densities of the three branches. However, since only one of the overlapping densities extends to $\theta_{0}$, the density diverges at $\theta_{0}$ as $\left(\theta-\theta_{0}\right)^{-1 / 2}$.

For $M \geq 3$ the polynomial equation (26) can be solved only numerically. We have carried out its numerical solution for $3 \geq M \geq 8$ and determined the corresponding values of $\theta_{0}$. The result of $M=3, N=400$ is shown in Fig. 4. We also show in Fig. 5 the trend of $\theta_{0} \rightarrow 0$ as $M \rightarrow \infty$.

In the limit of $M \rightarrow \infty$, we note that for $M$ large we must have $\left|e^{t(\phi)}\right|=1$. This is so since, otherwise, without the loss of generality we can assume $\left|e^{t(\phi)}\right|>1$ and (25) yields $s \sim e^{-t(\phi)}$ which contradicts (20) (except at $\phi=\pi$ ). Thus we write $t(\phi)=i \alpha(\phi)$ where $\alpha(\phi)$ is real. Then, (20) becomes

$$
s+s^{-1}=2 \cos \alpha+2(1+\cos \phi)=\text { real }
$$

leading to the bound $-2 \leq s+s^{-1} \leq 6$. For $-2 \leq s+s^{-1} \leq 2$ we must have $|s|=1$ which implies, by Theorem 2(iii), that all zeroes are on the circle $|x+\sqrt{2}|=1$. For $2 \leq s+s^{-1} \leq 6$, the bound is equivalent to the bound (33) on $s$ and, by Theorem 1(ii), all zeroes are on the unit circle $|x|=1$. We therefore conclude that in the limit of $M \rightarrow \infty$ all zeroes are on the two circles $|x|=1$ and $|x+\sqrt{2}|=1$. Indeed, this coincides with the finding of Fisher [2] who showed that, in the limit of both $M, N \rightarrow \infty$, the partition function zeroes are on the two equivalent circles $|\tanh K \pm 1|=\sqrt{2}$ [13]. However, our result is more general and holds for $M \rightarrow \infty$ and any $N$.

Finally, we remark that our results confirm the conjecture put forth in [5], that all zeroes in the $\operatorname{Re}(x)>0$ half plane are on the unit circle $|x|=1$. But a generally proof of this conjecture is still lacking at this point.

\section{DISCUSSIONS}

We have obtained the closed-form expression of the partition function of an $M \times N$ rectangular Ising lattice with an asymmetric boundary condition, and evaluated its zeroes. It was found that the zeroes are given by the roots of the polynomial equation (26). Particularly, we established that real roots of (26) lead to zeroes either on the unit circle $|x|=1$ or within the segment $[-(\sqrt{2}+1),-(\sqrt{2}-1)]$ of the negative real axis in the complex $x$ plane. We also established that all zeroes lie on two unit circles centered respectively at

$x=0,-\sqrt{2}$ in the $M \rightarrow \infty$ limit for any $N$, where $x=\left(e^{2 K}-1\right) / \sqrt{2}$. Numerical results for finite $M$ are also presented.

\section{ACKNOWLEDGEMENT}

Work has been supported in part by National Science Foundation Grant DMR-9614170. 


\section{APPENDIX. EVALUATION OF A PFAFFIAN}

For completeness we include in this appendix an outline of the steps leading to expressing the graph generating function $G_{\mathcal{L}^{*}}(z)$ as a Pfaffian. First, following standard procedures [8] we see that the graph generating function $G_{\mathcal{L}^{*}}(z)$ for $\mathcal{L}^{*}$ is equal to the dimer generating function for a "bathroom-tile" lattice constructed from $\mathcal{L}^{*}$ as shown in Fig. 6. This leads to the relation

$$
G_{\mathcal{L}^{*}}=\operatorname{Pf} A=\sqrt{\operatorname{det}|A|}
$$

where $\operatorname{Pf} A$ is the Pfaffian of a $4(M+1) N \times 4(M+1) N$ antisymmetric matrix

$$
A=A_{M} \otimes I_{N}+C_{M} \otimes H_{N}-C_{M}^{T} \otimes H_{N}^{T} .
$$

Here, $I_{N}$ is the $N \times N$ identity matrix, $H_{N}$ is an $N \times N$ matrix given by

$$
H_{N}=\left(\begin{array}{ccccc}
0 & 1 & 0 & \ldots & 0 \\
0 & 0 & 1 & \ldots & 0 \\
\vdots & \vdots & \vdots & \ddots & \vdots \\
0 & 0 & 0 & \ldots & 1 \\
-1 & 0 & 0 & \ldots & 0
\end{array}\right)
$$

$A_{M}$ and $C_{M}$ are $4(M+1) \times 4(M+1)$ matrices given by

$$
\begin{aligned}
& C_{M}=\left(\begin{array}{ccccc}
C(z) & 0 & & & \\
0 & C(z) & & & \\
\vdots & \vdots & \ddots & \vdots & \vdots \\
& & & C(z) & 0 \\
& & & 0 & C(1)
\end{array}\right) \\
& A_{M}=\left(\begin{array}{ccccc}
B(0) & B_{+}(z) & & & \\
B_{-}(z) & B(0) & B_{+}(z) & & \\
\vdots & \vdots & \ddots & \vdots & \vdots \\
& & B_{-}(z) & B(0) & B_{+}(z) \\
& & & B_{-}(z) & B(0)
\end{array}\right)
\end{aligned}
$$

with

$$
\begin{aligned}
C(z)=\left(\begin{array}{cccc}
0 & 0 & 0 & 0 \\
0 & 0 & 0 & 0 \\
0 & 0 & 0 & z \\
0 & 0 & 0 & 0
\end{array}\right), & B(z)=\left(\begin{array}{ccccc}
0 & 1 & -1 & -1 \\
-1 & 0 & 1 & -1 \\
1 & -1 & 0 & 1+z e^{i \phi} \\
1 & 1 & -\left(1+z e^{-i \phi}\right) & 0
\end{array}\right), \\
B_{+}(z)=\left(\begin{array}{cccc}
0 & z & 0 & 0 \\
0 & 0 & 0 & 0 \\
0 & 0 & 0 & 0 \\
0 & 0 & 0 & 0
\end{array}\right), & B_{-}(z)=\left(\begin{array}{cccc}
0 & 0 & 0 & 0 \\
-z & 0 & 0 & 0 \\
0 & 0 & 0 & 0 \\
0 & 0 & 0 & 0
\end{array}\right) .
\end{aligned}
$$

Here $H_{N}^{T}$ and $C_{M}^{T}$ denote, respectively, the transpose of $H_{N}$ and $C_{M}$.

Now, $H_{N}$ and $H_{N}^{T}$ can be diagonalized simultaneously with respective eigenvalues $e^{i \phi}$ and $e^{-i \phi}$, where $\phi=\pi(2 n-1) / N, n=1,2, \cdots, N$. We can therefore replace $H_{N}\left(\right.$ resp. $\left.H_{N}^{T}\right)$ by a diagonal matrix with diagonal elements $e^{i \phi}\left(\operatorname{resp} . e^{-i \phi}\right)$ without affecting the determinant. The substitution of (A2) into (A1) now leads to (i) 


\section{Figure Captions}

Fig. 1. (a) A $3 \times 8$ self-dual lattice $\mathcal{L}$. (b) The $4 \times 8$ lattice $\mathcal{L}^{*}$ derived from $\mathcal{L}$.

Fig. 2. Loci of zeroes in the $x$ plane for $M=1, N=4,000$.

Fig. 3. Loci of zeroes in the $x$ plane for $M=2, N=2,000$.

Fig. 4. Loci of zeroes in the $x$ plane for $M=3, N=400$.

Fig. 5. The dependence of $\theta_{0}$ on $M$.

Fig. 6. The bathroom-tile lattice for a $3 \times 4$ lattice $\mathcal{L}^{*}$. 


\section{REFERENCES}

[1] C. N. Yang and T. D. Lee, Phys. Rev. 87, 404 (1952).

[2] M. E. Fisher, in Lecture Notes in theoretical Physics, edited by W. E. Brittin (University of Colorado Press, Boulder, 1965), Vol. 7c.

[3] Historically, a systemic study of the zeroes of the Ising partition function in the thermodynamic limit was first carried by Stephenson. See, J. Stephenson, Physica 148A, 107 (1988), and references cited therein.

[4] More recently, zeroes of the Potts model including those of the Ising model have been studied extensively by R. Shrock and co-workers using enumerations techniques and low-temperature expansions. See, V. Matveev and R. Shrock, Phys. Rev. E 54, 6174 (1996) and references cited therein.

[5] For a numerical study of the Potts zeroes, see C. N. Chen, C. K. Hu, and F. Y. Wu, Phys. Rev. lett. 76, 169 (1996), and references cited therein.

[6] Z. Glumac and K. Uzelac, J. Phys. A 27, 7709 (1994), and F. Y. Wu, to be published.

[7] Eq. (3) follows from the fact that to each closed polygonal configuration in $\mathcal{L}$ there exist two closed polygonal configurations in $\mathcal{L}^{*}$, obtained by completing the polygons along the innermost circle in two different ways. These two configurations carry the same weight since the edge weight for the innermost circle is 1 .

[8] P. W. Kasteleyn, Physica 27, 1209 (1961).

[9] B. M McCoy and T. T. Wu, The Two-dimensional Ising Model, (Harvard University Press, 1973).

[10] B. M McCoy and T. T. Wu, Phys. Rev. 162, 436 (1967).

[11] See, for example, I. S. Gradshteyn and I. M. Ryzhik, Tables of Integrals, Series, and Products, (Academic Press, New York, 1965), p. 34, 1.394 with $x=y=1$ and $a=-\pi / n$.

[12] F. Y. Wu, G. Rollet, H. Y. Huang, J. M. Maillard, C. K. Hu, and C. N. Chen, Phys. Rev. 76, 173 (1996).

[13] Note that in comparing the two results, the caption of Fig. 19.1 of [2] contains a misprint where $v=\tanh (K / 2)$ should read $v=\tanh K$. 


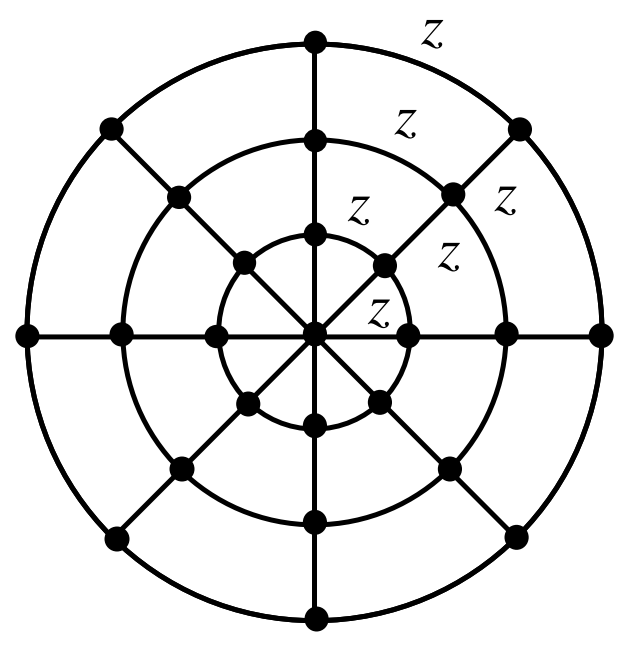

(a)

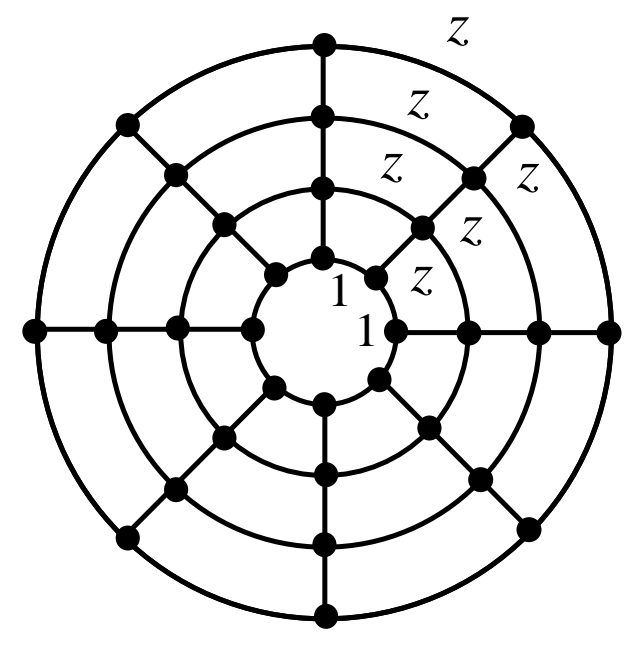

(b)

Fig.1 


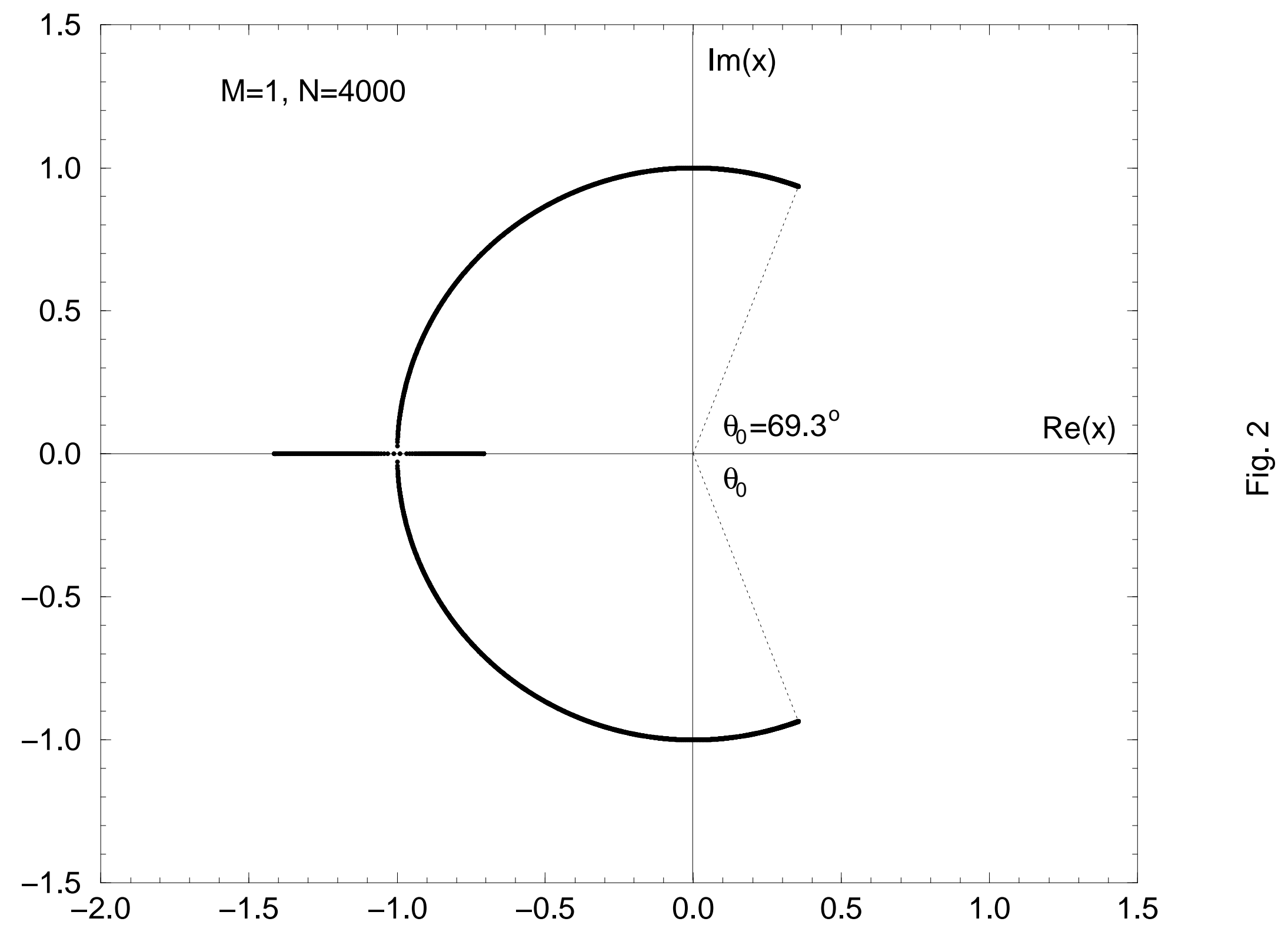




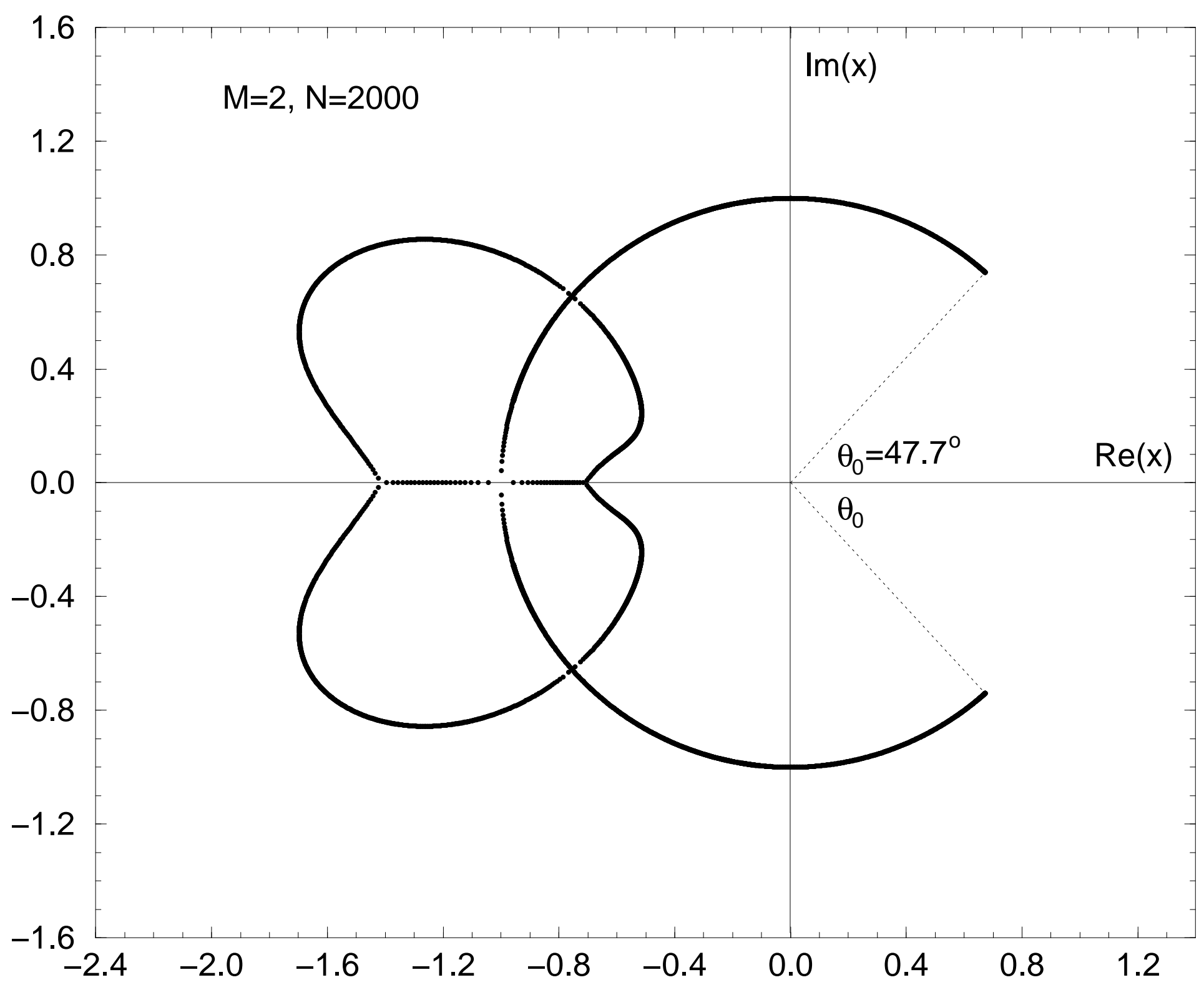

몬 


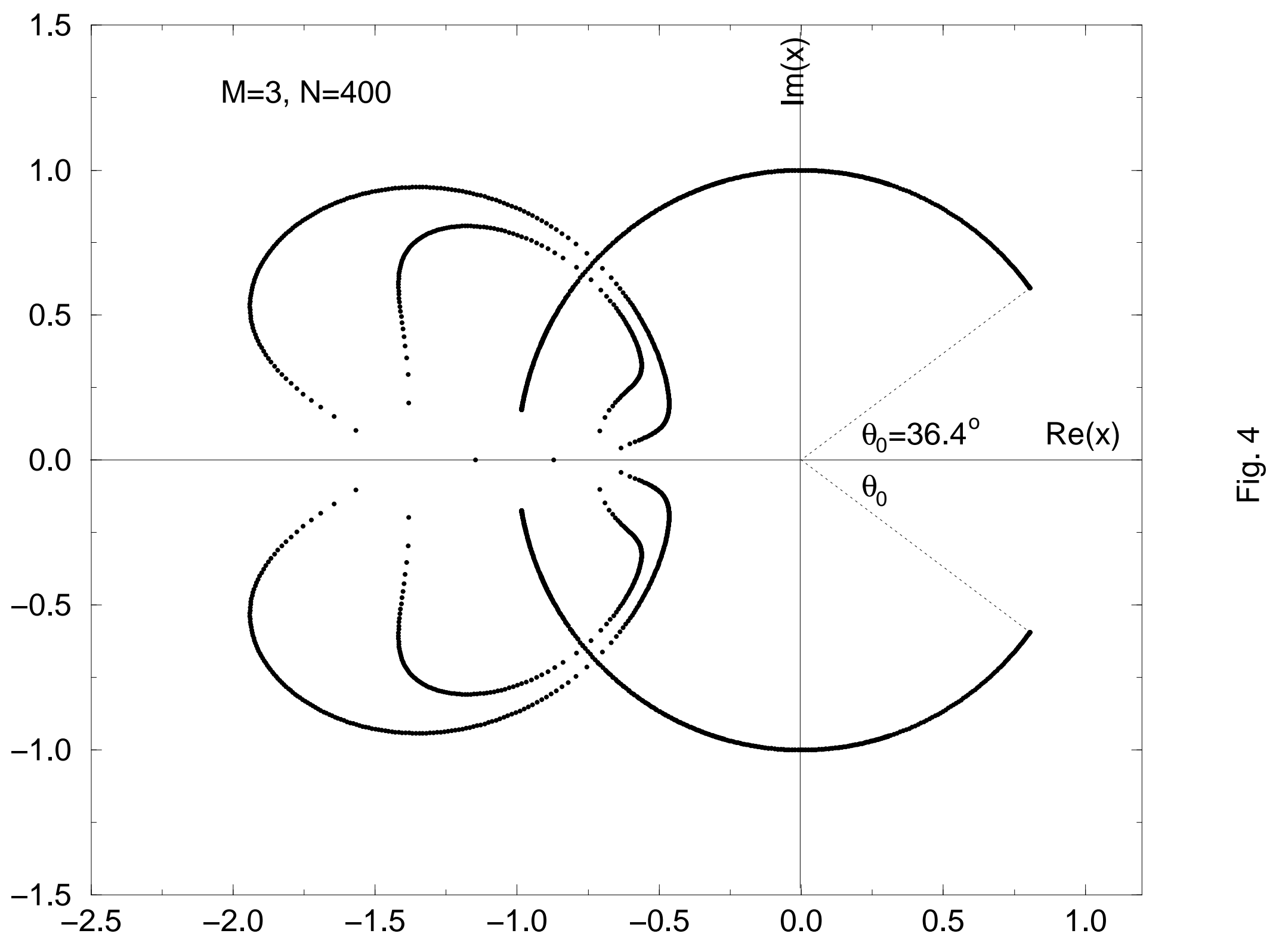




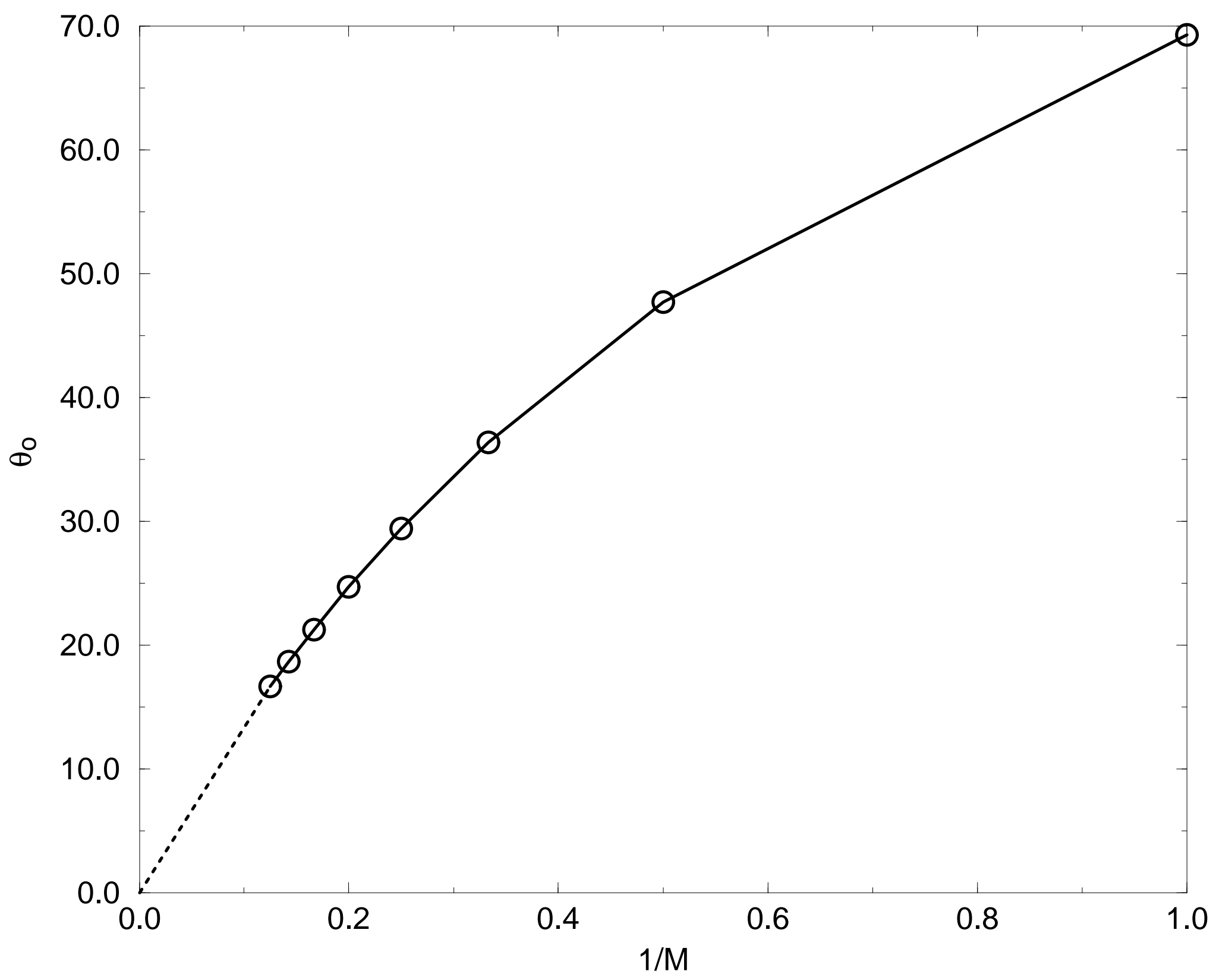

옴 


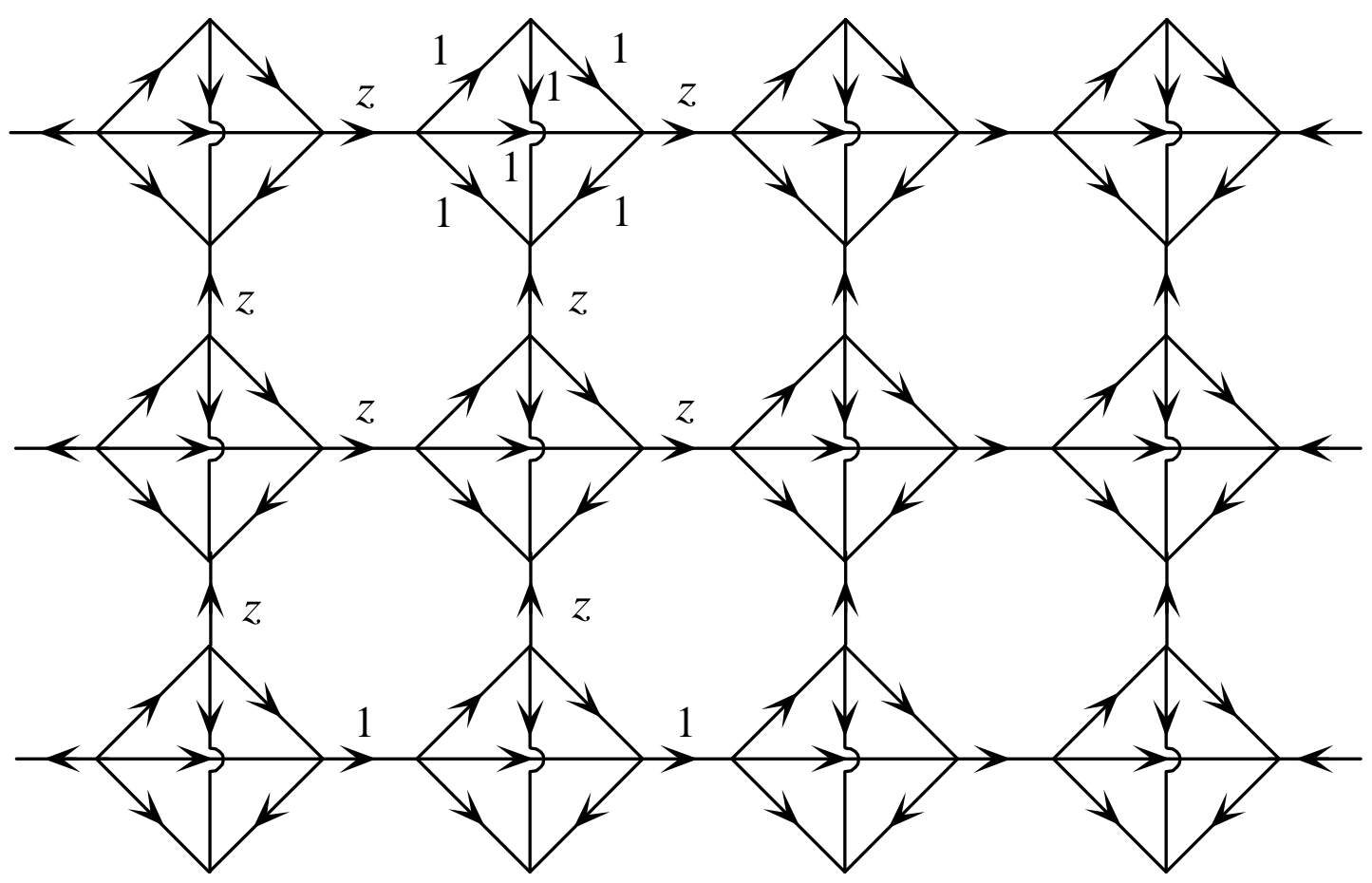

Fig. 6 